\title{
An $n$-back task using vibrotactile stimulation with comparison to an auditory analogue
}

\author{
ROBERTA L. KIATZKY \\ Carnegie Mellon University, Pittsburgh, Pennsylvania \\ AND \\ Nicholas A. Giudice, James R. MARSton, Jerome Tietz, \\ REGINALd G. GolledGe, AND JACK M. LOOMIS \\ University of California, Santa Barbara, California
}

\begin{abstract}
We report a vibrotactile version of the common $n$-back task used to study working memory. Subjects wore vibrotactile stimulators on three fingers of one hand, and they responded by pressing a button with the other hand whenever the current finger matched the one stimulated $n$ items back. Experiment 1 showed a steep decline in performance as $n$ increased from 1 to 3; each additional level of $n$ decreased performance by $1.5 d^{\prime}$ units on average. Experiment 2 supported a central capacity locus for the vibrotactile task by showing that it correlated strongly with an auditory analogue; both tasks were also related to standard digit span. The vibrotactile version of $n$-back may be particularly useful in dual-task contexts. It allows the assessment of cognitive capacity in sensory-impaired populations in which touch remains intact, and it may find use in brain-imaging studies in which vibrotactile stimuli impose a memory load.
\end{abstract}

A widely used means of measuring working memory is the $n$-back task (see below for a review). In this paradigm, a series of stimuli are presented, and the subject must signal whenever the current stimulus matches the one that was presented $n$ steps back in the list. Variations in $n$ can be used to assess individuals' levels of processing capacity or to set a level of dual-task competition.

Since its inception, the $n$-back task has been brought to bear'on a host of issues irelated to working memory. Early work with the task established its use for investigating working memory at a cortical level. Braver and associates (Braver et al., 1997) showed that areas of prefrontal cortex were increasingly activated as the $n$-back interval grew longer. Jonides and associates (Jonides et al., 1997) examined 11 different areas associated with verbal working memory and found that most showed increases in activation with $n$-back load, whereas, conversely, other areas not associated with working memory from behavioral tasks were unaffected by load. Cohen and associates (Cohen et al., 1997) traced the effect of the $n$-back load on cortical activation to processing intensity, as distinct from processing time.

The $n$-back paradigm has been used to model individual differences in working memory capacity (Lovett, Daily, \& Reder, 2000). It has also seen widespread use to measure working memory capacity in special populations, such as drug abusers (Verdejo-Garcia, Bechara, Recknor, \& PerezGarcia, 2006), traumatic-brain-injured patients (McAllister et al., 1999), and schizophrenics (Callicott et al.,
2003). The task has been invoked in neuropsychological studies of patients showing specific deficits (Ravizza, Behrmann, \& Fiez, 2005). Modality-specific variations of the paradigm have been devised. For example, Postle, D'Esposito, and Corkin (2005) introduced different types of distractors for different classes of $n$-back targets; they found that verbal distraction affected $n$-back performance with objects, whereas motion distraction affected performance with object location.

For the present purposes, it is noteworthy that visual stimuli predominate in the $n$-back literature. Owen, McMillan, Laird, and Bullmore (2005) reported a metaanalysis of 24 studies using the $n$-back task in conjunction with brain imaging. Most were visual, about half involving verbal stimuli and the others using shapes, faces, and pictures. One study used odors. None, however, invoked the modality of touch.

We developed a tactile version of the $n$-back task to assess the cognitive load of different forms of navigation guidance for the blind (Klatzky, Marston, Giudice, Golledge, \& Loomis, 2006). In a long-standing program of study, we have asked whether people attempting to walk to target locations without vision can be successfully guided by correction signals in the form of virtual (spatialized) sound (i.e., the target appears perceptually to emanate from a point of space, to which the navigator "homes") or spatial language (i.e., corrective signals such as "left" or "left 80 degrees"). In a number of stud- 
ies comparing guidance from virtual sound versus spatial language, we found a tendency for a small advantage for virtual sound with respect to path-following performance and preference (Loomis, Golledge, \& Klatzky, 1998; Loomis, Marston, Golledge, \& Klatzky, 2005; Marston, Loomis, Klatzky, Golledge, \& Smith, 2006). We then asked whether a more robust difference would be found with respect to cognitive load (Klatzky et al., 2006). We hypothesized that the direct perceptual signal from virtual sound would impose a lower load on working memory than language-based commands. Because hearing was involved in the navigation guidance and our subjects were denied vision, we developed a version of the $n$-back task using vibrotactile stimulation, in which the identity of the stimulated finger became the stimulus to match. As we predicted, when performing navigation concurrent with a 1-back load, subjects guided by language navigated more slowly and deviated more from the correct path than did those guided by virtual sound. No such differences were found in the absence of the $n$-back load.

In Experiment 1, we report the general method for the vibrotactile $n$-back task, along with data from conditions where $n$ varies from 1 to 3 . Subjects wore vibrotactile stimulators on three fingers of one hand, and they were instructed to respond by pressing a button with the other hand whenever the current finger matched the one stimulated $n$ items back. To avoid vibrotactile adaptation, the hands were switched between blocks of vibratory pulses. We chose a frequency of $290 \mathrm{~Hz}$, which was near the peak frequency of the vibrator and should optimally activate the deep mechanoreceptors, the pacinian corpuscles (Bolanowski, Gescheider, Verrillo, \& Checkosky, 1988). Experiment 2 compared the vibrotactile task with an auditory analogue using spatially localized tones of the same frequency used for vibrotaction.

Both accuracy and reaction time (RT) for successful matches are commonly used in conjunction with $n$-back tasks. Accuracy is sometimes reported as an overall measure (see, e.g., Lovett et al., 2000), or it can be broken down into the signal detection partitioning into proportions of hits and false alarms, yielding $d^{\prime}$ or an alternative statistic (e.g., Mohr \& Linden, 2005; Ragland et al., 2002). Typically, accuracy declines and RT increases with increasing levels of $n$. Using letters as stimuli, with $n=1$, 2 , or 3, McElree (2001) implemented a modified $n$-back task with letter stimuli in conjunction with a responsecuing paradigm, in order to directly assess the relation between RT and accuracy $\left(d^{\prime}\right)$. The author reported that $d^{\prime}$ increased with the processing time (i.e., cue lag plus response latency) up to about $1 \mathrm{sec}$, at which point $d^{\prime}$ reached ceiling. For a given value of processing time, $d^{\prime}$ declined with increasing $n$. Moreover, the higher the value of $n$, the more slowly $d^{\prime}$ improved given longer processing time. This indicates that in the conventional task, the two measures are largely redundant. RT presents two other considerations worth noting. First, the duration of the interstimulus interval can bias the observed RTs by enforcing a cutoff for long latency values; second, the RT will generally be based on fewer responses as $\boldsymbol{n}$ increases and the proportion of hits decreases.

In the present study, given the temporally extended nature of the vibrotactile stimuli, together with the foregoing considerations, we chose to measure only accuracy, in the form of hits and correct rejections ( $=1-$ false alarms). Pilot testing indicated that we would have a relatively small number of responses on which to base RT with higher $n$ values.

\section{EXPERIMENT 1}

\section{Method}

Subjects. Sixteen students from the UCSB population were recruited through word of mouth and e-mails, ranging in age from 19 to 23 years $(S D 1.08)$ and evenly distributed by gender.

Apparatus. The stimulators for the $n$-back task were three vibrators (VBW32 Skin Transducer-Audiological Engineering, Somerville, MA; $2.54 \mathrm{~cm}$ long $\times 1.85 \mathrm{~cm}$ wide $\times 1.07 \mathrm{~cm}$ thick) mounted with Velcro on the tip of the subject's thumb, middle finger, and little finger (Figure 1). Vibration on the finger was controlled by the computer (Model ES100; Gateway, Irvine, CA). A vibration was initiated by using a sound card (Montego card; Voyetra Turtle Beach, Yonkers, NY) to create a $290-\mathrm{Hz}$ square wave WAV file and routing the audio signal from the speaker output of the card to one of the vibrators by means of an Advantech PCL-812PG (Milpitas, CA) multi-input-output ( $(/ O)$ card that was connected to a customdesigned multiplexer circuit. The circuit consisted of three relays that were opened or closed by connecting the relay coils to the three digital $\mathrm{I} / \mathrm{O}$ pins of the multi-I/O card. Software commands issued from within the main application, created with Vizard software (WorldViz, Santa Barbara, CA: www.worldviz.com), turned each relay on or off depending on which vibrator was signaled on a particular trial. The handheld response button, a momentary-contact push-button switch, was connected to another digital $\mathrm{I} / \mathrm{O}$ pin in order to monitor the subject's responses.

Vibrations were activated for $0.5 \mathrm{sec}$ and were followed by a 1 -sec interval, during which the subject was to respond if a match was detected. The choice of finger to stimulate was specified by a randomly generated series of the integers 1,2 , and 3, resulting in a probability of an $n$-back repeat equal to $1 / 3$ for each level of $n$.

Procedure. Subjects were instructed to depress the push button whenever a vibrotactile stimulus was received by the same finger that had been stimulated $n$ steps back. The computer recorded a correct identification (hit) if a match was present and the response button was pressed within the $1-\mathrm{sec}$ intervibration interval. Failures to respond were recorded as misses. Responses on nonmatching trials were recorded as false alarms; otherwise, the nonresponse was counted as a correct rejection.

Prior to the experimental trials, the $n$-back task was explained with verbal examples and by tapping the subject's fingers. The vibrators were then attached to the hand, and a sample series of 11 pulses was given, constrained so that it exemplified three 1-backs, three 2-backs, and four 3-backs. Subjects were then blindfolded, and a baseline test was conducted for $1 \mathrm{~min}$ in the 1-back mode. Our intention was to eliminate subjects who failed to achieve at least $80 \%$ hits and $80 \%$ correct rejections in this test, but all passed.

Subjects took part in three blocks of experimental trials, each block containing one sequence with $n=1$, one with $n=2$, and one with $n=3$. The order of $n$ values was kept constant across blocks and counterbalanced by a Latin square across subjects as equally as possible. For a given $n$ value within a block, there was a sequence of $100+n$ consecutive vibrations, lasting about $2.5 \mathrm{~min}$. Only the last 100 trials were analyzed, since the first $n$ were used to allow matches on all of the subsequent trials (e.g., with $n=1$, the first 


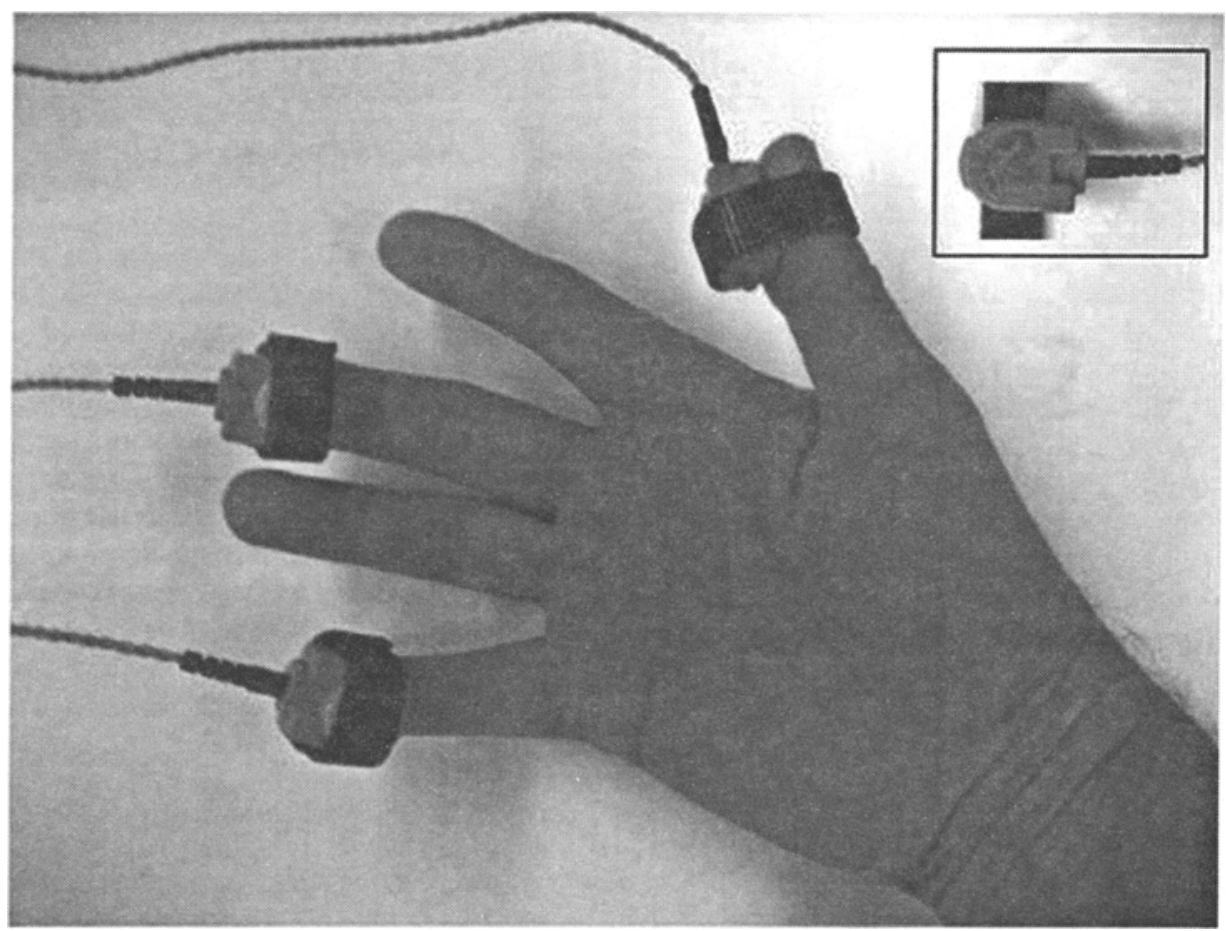

Figure 1. Placement of vibrators on fingers; inset shows a single vibrator.

possible match is on Trial 2). To reduce vibrotactile adaptation, the hand receiving stimulation was alternated between blocks, with half of the subjects beginning with the dominant hand (by self-report) and half with the nondominant hand. The other hand then held the push button for the response.

\section{Results and Discussion}

An initial examination of the data by block indicated essentially invariant performance across blocks, for all levels of $\boldsymbol{n}$. There was no evidence of a decline over blocks, as might be expected if there had been sensory adaptation to the vibrators (proportion correct was actually slightly greater for Block 3 than for Block 1, by .02 on average for hits and .01 for correct rejections). There was also no evidence of learning over blocks for any $n$. Data were therefore pooled across blocks for analysis.

Figure 2 shows the hit rate and correct rejection rate for each level of $n(1,2,3)$. The effect of $n$ was significant in one-way repeated measures ANOVAs on each variable $[F(2,26)=120.31$ for hits and 39.43 for correct rejections, $p s<.001]$. It is clear from the figure that the greatest effect of increasing $n$ was to decrease the hit rate, with correct rejections declining relatively little. The $d$ 's, as computed from the mean values over subjects, were $3.87,2.13$, and 0.89 for $n$ values of 1,2 , and 3, respectively. Thus, an increase of 1 in $n$ produced an average drop of about $1.5 d^{\prime}$ units.

The probability of a hit by chance depends both on the overall rate of pressing the button and the probability of an $n$-back match. Given that hits were possible on $1 / 3$ of trials, the buttonpress rate was calculated for a given $n$ as proportion hits $\times 1 / 3+$ proportion false alarms $\times$ $2 / 3$ - which decreased with $n$. The probability of an $n$-back match was $1 / 3$ for all $n$. The chance hit rates, which were the products of these values, were $10 \%, 9 \%$, and $7 \%$ for $n$ values of 1,2 , and 3 . Thus, the hit rate remained well above chance for all values of $n$.

In short, Experiment 1 clearly demonstrates an effect of working memory load in a vibrotactile version of the $n$-back task. A question raised by this study is whether the capacity demands of this task are unique to the modality of touch or are more general, and hence are tapped by other modalities. Experiment 2 addressed this issue.

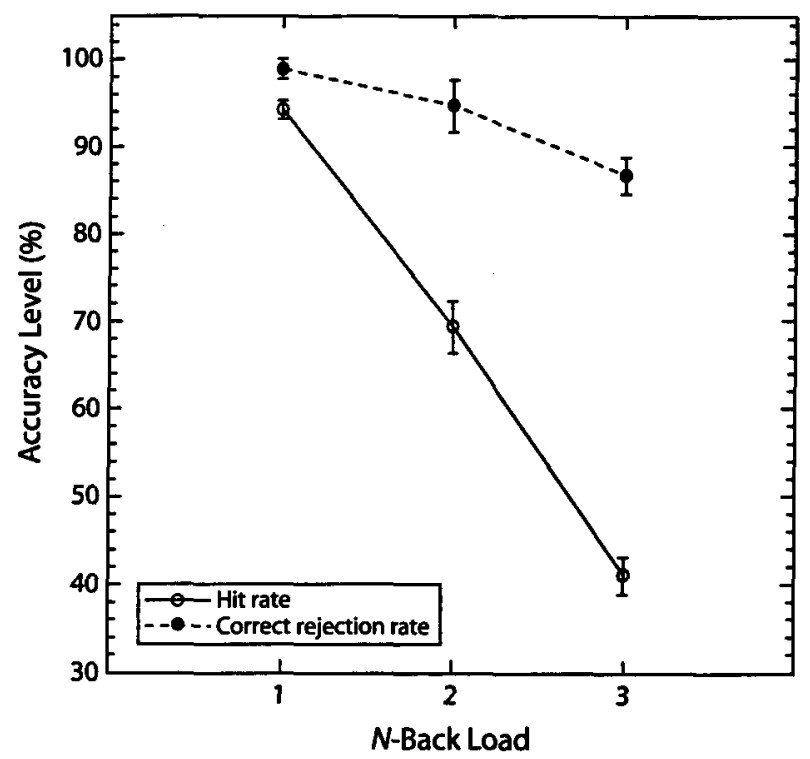

Figure 2. Hit rate and correct rejection rate by value of $\boldsymbol{n}$ in Experiment 1. Error bars are 1 SEM. 


\section{EXPERIMENT 2}

Experiment 2 investigated whether the effect of $n$-back load in the vibrotactile modality reflected a central capacity limitation shared across modalities. It assessed similarity of outcomes between the vibrotactile task and a directly analogous task using auditory stimuli. Previous research on the $n$-back task in the auditory modality had used a variety of stimuli, including spoken letters and digits, words varying in pitch, frequency-modulated tones, and-importantly for present purposes-tones varying in location (see Owen et al., 2005, for a review). Experiment 2 used an $n$-back task requiring matching the spatial locations of auditory stimuli; this task was compared with a vibrotactile version, as used in Experiment 1. The auditory task was based on one designed by Martinkauppi and colleagues (Martinkauppi, Rama, Aronen, Korvenoja, \& Carlson, 2000), in which subjects matched tones presented to the left, center, or right. Thus, as in the vibrotactile task, there were three possible locations, which were matched at intervals of $n=1,2$, and 3. If the two modalities tapped a common capacity, they should show a similar fall-off with $\boldsymbol{n}$. One would also expect correlations between modalities across subjects, reflecting individual differences in the capacity shared by the two tasks. As an additional measure of individuals' working memory, we administered a backward digit span task.

\section{Method}

Subjects. Twelve students were recruited from the UCSB population, ranging in age from 19 to 25 years $(S D 1.53)$ and evenly distributed by gender. All were right-handed. Subjects took part in a 1-min pretest in both the auditory and vibrotactile modalities to ensure that they had normal tactile/audio acuity and could perform at a baseline level of at least $\mathbf{8 0 \%}$ hits and $80 \%$ correct rejections with $n=1$, as in Experiment 1.

Apparatus. The procedure for the vibrotactile $n$-back task was identical to that in Experiment 1, except that, since there was only one block of vibrotactile trials, only the nondominant hand was exposed to the vibrotactile stimulus.

For the auditory $n$-back task, subjects wore closed-cup headphones (Realistic Nova 40, RadioShack). They monitored three positions of an audio tone-left, right, or central-relative to the ear axis. Identically to the vibrotactile task, the signal was a $290-\mathrm{Hz}$ square wave tone, in this case routed to the headphones rather than the vibrators. The tone was on for $0.5 \mathrm{sec}$ and followed by $1 \mathrm{sec}$ of silence, during which the subject responded if a location match at the requisite value of $n$ was detected. The response was made by pressing a button in the dominant hand.

For the auditory signal, three separate wave files were made, each $0.5 \mathrm{sec}$ in duration, to be played back through the sound card of the experiment computer. With the center tone, both channels of the stereo wave were preserved and amplitude-equalized. For the left and right stimuli, the nondesired channel of the stereo wave was deleted. The perceived volume of the three tones was equated by the experimenters using subjective matching.

Procedure. Each subject took part in a block of trials with each modality, within which there were trials with $n$ set to 1,2 , and 3 , for a total of six trials. The order of $n$ within a block and the modality order were counterbalanced. Both modalities had the same match probability of $1 / 3$ for each level of $n$.

The task was initially briefly explained for each modality. Prior to a new value of $n$, the subject practiced with a brief, predetermined sequence with feedback, in which three matches were presented for 1 and 2 back and four matches for 3 back.
Upon conclusion of the six $n$-back trials, subjects performed a backward digit span test of working memory. They were instructed to repeat, in reverse order, a sequence of digits, which were presented as wave files through the headphones with a 1 -sec interstimulus interval. Beginning with two digits, two trials at each sequence length were given, and if at least one was correct, the sequence length was incremented, until both trials produced errors. A fixed sequence of digits was used for all subjects, presented in a pseudorandomized order, such that numbers did not repeat or appear sequentially-for example, 2,2 or $1,2,3$.

\section{Results and Discussion}

The average hit and correct rejection rates by modality and $n$ are shown in Figure 3. Given numerous perfect scores ( $100 \%$ hits or correct rejections) in the $n=1$ condition, and three trials without errors in the $n=2$ condition, we chose to examine $d^{\prime}$ computed over the averaged scores for $n=2$ and $n=3$.

An ANOVA on percent hits produced only an effect of $n$ $[F(2,22)=121.98, p<.001]$. The effect of modality was not significant $[F(1,11)=3.56, p=.09]$, and the interaction did not reach significance either $[F(2,22)=2.77, p=$ .08 ]. (The power for the interaction was .49 , suggesting that a larger sample might prove it significant, but the effect size was not large; $\eta_{\mathrm{p}}^{2}=.20$.) The corresponding ANOVA on correct rejections had parallel effects: for $n[F(2,22)=$ $47.16, p<.001]$, for modality $[F(11),=4.36, p=.06]$, and for the interaction $[F(2,22)<1]$. Although the modality effects in the ANOVAs did not reach significance, the difference between modalities with respect to $d^{\prime}$, based on the $n=2$ and 3 average $\left(d^{\prime}=1.65\right.$ for auditory vs. 1.35 for vibrotactile), was significant $[t(11)=2.33, p<.05]$, indicating a small advantage for the auditory condition in overall target detection. This may reflect greater spatial separation for the tones than for the finger vibrations.

Most important are the findings that the effect of $n$ was highly similar across modalities, and there were strong

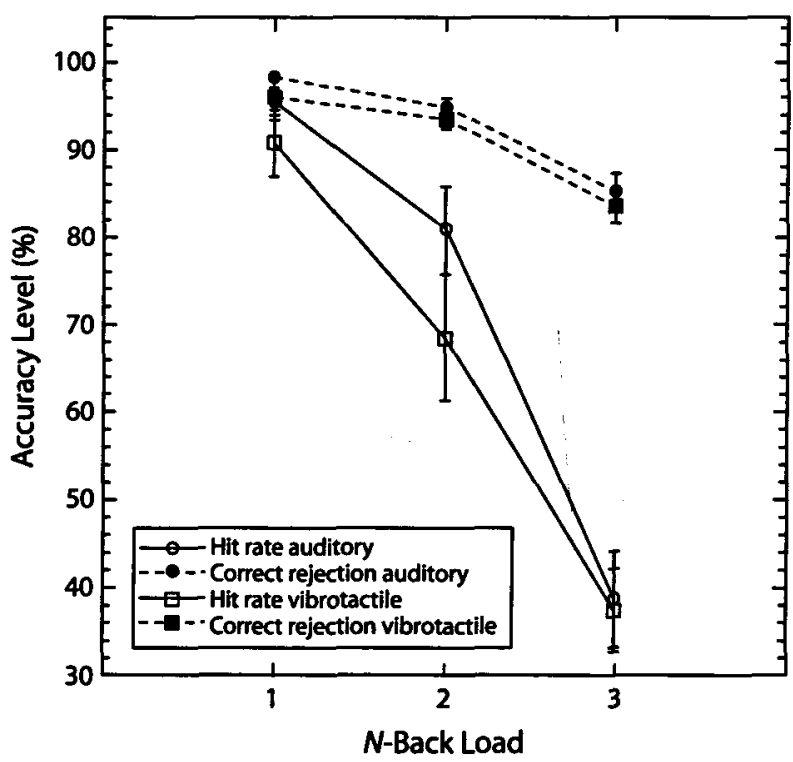

Figure 3. Hit rate and correct rejection rate by modality and value of $\boldsymbol{n}$ in Experiment 2. Error bars are 1 SEM. 
Table 1

Correlations Between the Auditory and Vibrotactile $N$-Back Tasks $(d f=10$, all $p s<.05)$

\begin{tabular}{ll}
\hline \multicolumn{1}{c}{ Variable } & $r$ \\
\hline$\%$ hits & .75 \\
$\%$ correct rejections & .79 \\
$d^{\prime}$ for $n=2$ and 3 & .68 \\
\hline
\end{tabular}

correlations across subjects between the auditory and vibrotactile conditions, shown in Table 1 . These findings clearly indicate common sources of variance, independent of modality, and support the interpretation of the vibrotactile $n$-back task as a measure of a central capacity limitation.

The backward digit span task produced 3 subjects with spans of 3,7 with spans of 4, and 2 with spans of 5 . This small range limits the usefulness of the correlations. Figure 4 groups subjects according to digit span and then reports their mean accuracy for the six conditions of the experiment. There was a tendency for performance in both modality conditions to improve with span, except for $n=1$, when performance was at ceiling. A larger sample of subjects would be needed to systematically explore the relation of the $n$-back tasks to the memory span, but these results suggest a tendency for $n$-back performance in both modalities to improve with span.

\section{GENERAL DISCUSSION}

We report an extension of the well-known $n$-back paradigm, used to assess working memory, to the vibrotactile modality. Accuracy doclines regularly with $n$, as has been found for other versions of the task. The effects of $n$ with the 'vibrotactile task are very similar to those with a directly analogous auditory task. Moreover, performance in

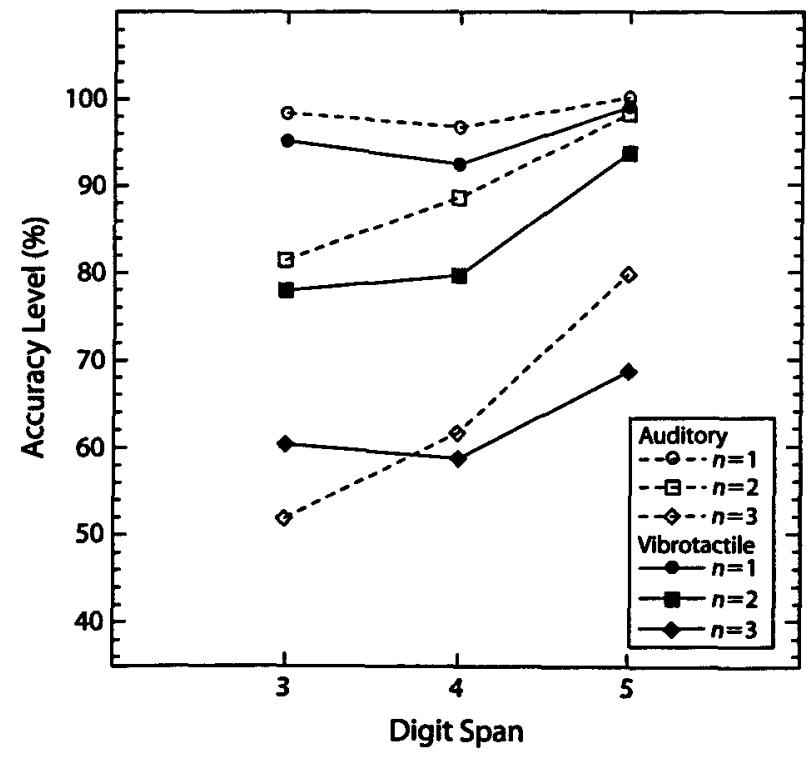

Figure 4. Average overall accuracy in Experiment 2 for groups of subjects with each level of digit span, by modality (auditory, vibrotactile) and value of $n$. the two modalities is highly correlated, and at least tentatively, both appear to vary with working memory as measured by digit span. These data, though based on a small number of subjects, suggest that the vibrotactile task taps a central memory capacity shared with other modalities.

In addition to accuracy data, one could, if desired, include RT as a measure with the present paradigm, using the onset of the vibratory signal to start the latency clock. The time to respond to a vibratory signal has been shown to be modulated by attention (see Spence, Pavani, Maravita, \& Holmes, 2004, for a review), suggesting that effects of the $n$-back manipulation on RT could be found within this modality. However, given that vibration thresholds vary with frequency and amplitude (Verrillo, 1963), this is feasible only if the vibratory profile is held constant across the experiment.

The vibrotactile version of the $n$-back task potentially has broad utility. First, it allows the assessment of cognitive capacity in populations for which other modalities are impaired but touch remains intact. Using touch may be particularly advantageous when dual-task interactions are studied, because it provides an additional modality for the competing-load task. Thus, for example, blind people can be tested with auditory cues and touch, and deaf with visual cues and touch. Our development of the vibrotactile $n$-back task resulted, in fact, from our interest in the cognitive load on blind individuals when they attempted to navigate with the aid of auditory cues. The results of our previous study (Klatzky et al., 2006) confirmed the viability of the task to provide a competing load with spatial language.

The method also points to the possibility of brainimaging studies in which vibrotactile stimuli impose a memory load. Such studies are abundant with visual stimuli, as we have noted. Importantly, vibratory stimulators could be applied to skin areas remote from the head, potentially reducing interference with devices mounted around the head.

The adaptation of the $n$-back task to touch may also lead to new studies that focus directly on this relatively understudied modality and its neural basis. Working memory capacity for vibratory stimuli can be assessed, for example, under parametric variations in the stimulus and its location in the skin. It would be interesting to compare memory capacity to the vibrotactile threshold of various skin sites to determine whether peripheral as well as central limitations play a role.

The nature of any limitations would depend to a great extent on how the stimulus locations are encoded. With only three stimuli to track, people might verbally code which finger is stimulated, or they might transform the finger identities into locations coded visuospatially. Both of these strategies would transcend the input modality and presumably tap working memory. To the extent that there is some degree of modality-specific coding subject to peripheral constraints, it would weaken both intermodal correlations and the relation of $n$-back performance to measures of central working memory like digit span.

Although some modality specificity may be present, the vibrotactile variant of $n$-back appears to tap into lim- 
ited central capacity by means of the relatively novel channel of touch. It is offered here as a useful addition to the arsenal of working memory measures, one that does not load the typical sensory channels and hence offers new possibilities for application.

\section{AUTHOR NOTE}

This research was supported by Grant SB020101 from the National Institute on Disability and Rehabilitation Research, awarded to Sendero Group (Michael May, principal investigator). The authors thank Masaki Miyanohara for his assistance with collecting and organizing the data. Correspondence relating to this article should be sent to R. L. Klatzky, Department of Psychology, Carnegie Mellon University, Pittsburgh, PA 15213 (e-mail: klatzky@cmu.edu).

\section{REFERENCES}

Bolanowski, S. J., Jr., Gescheider, G. A., Verrilo, R. T., \& CheKKOSKY, C. M. (1988). Four channels mediate the mechanical aspects of touch. Journal of the Acoustical Society of America, 84, 1680-1694.

Braver, T. S., Cohen, J. D., Nystrom, L., Jonides, J., Smtth, E. E., \& NoLl, D. C. (1997). A parametric study of prefrontal cortex involvement in human working memory. NeuroImage, 5, 49-62.

Callicott, J. H., Mattay, V. S., Verchinski, B. A., Marenco, S., Egan, M. F., \& Weinberger, D. R. (2003). Complexity of prefrontal cortical dysfunction in schizophrenia: More than up or down. American Journal of Psychiatry, 160, 2209-2215.

Cohen, J. D., Perlstein, W. M., Braver, T. S., NYStrom, L. E., Noll, D. C., JoNIDES, J., \& SMITH, E. E. (1997). Temporal dynamics of brain activation during a working memory task. Nature, 386, 604-608.

Jonides, J., Schumacher, E. H., SMith, E. E., LAUbER, E. J., AWH, E., Minoshima, S., \& KoEPPE, R. A. (1997). Verbal working memory load affects regional brain activation as measured by PET. Journal of Cognitive Neuroscience, 9, 462-475.

KLatzKy, R. L., Marston, J. R., Giudice, N. A., GolledGe, R. G., \& Loomis, J. M. (2006). Cognitive load of navigating without vision when guided by virtual sound versus spatial language. Journal of Experimental Psychology: Applied, 12, 223-232.

LOOMIS, J. M., GOLLEDGE, R. G., \& KLATZKY, R. L. (1998). Navigation system for the blind: Auditory display modes and guidance. Presence: Teleoperators \& Virtual Environments, 7, 193-203.

LoOMIS, J. M., MARSTON, J. R., GolledGe, R. G., \& KLATZXY, R. L. (2005). Personal guidance system for people with visual impairment: A comparison of spatial displays for route guidance. Journal of Visual Impairment \& Blindness, 99, 219-232.
LOVETT, M. C., DAILY, L. Z., \& REDER, L. M. (2000). A source activation theory of working memory: Cross-task prediction of performance in ACT-R. Cognitive Systems Research, 1, 99-118.

Marston, J. R., LoOMis, J., KLATZKY, R. L., Golledge, R. G., \& SmITH, E. L. (2006). Evaluation of spatial displays for navigation without sight. ACM Transactions on Applied Perception, 3, 110-124.

Martinkauppi, S., Rama, P., Aronen, H. J., Korvenoja, A., \& Carlson, S. (2000). Working memory of auditory localization. Cerebral Cortex, 10, 889-898.

McAllister, T. W., Saykin, A. J., Flashman, L. A., Sparling, M. B., JoHnson, S. C., GUERIN, S. J., ET AL. (1999). Brain activation during working memory 1 month after mild traumatic brain injury: A functional MRI study. Neumology, 53, 1300-1308.

McElReE, B. (2001). Working memory and focal attention. Journal of Experimental Psychology: Learning, Memory, \& Cognition, 27, 817-835.

MoHR, H. M., \& LINDEN, D. E. (2005). Separation of the systems for color and spatial manipulation in working memory revealed by a dualtask procedure. Journal of Cognitive Neuroscience, 17, 355-366.

OWEN, A. M., MCMILLAN, K. M., LAIRD, A. R., \& BullMORE, E. (2005). $N$-back working memory paradigm: A meta-analysis of normative functional neuroimaging studies. Human Brain Mapping, 25, 46-59.

Postle, B. R., D'Esposito, M., \& CoRKIN, S. (2005). Effects of verbal and nonverbal interference on spatial and object visual working memory. Memory \& Cognition, 33, 203-212.

Ragland, J. D., Turetsky, B. I., GuR, R. C., Gunning-Dixon, F., TURner, T., SCHROEDER, L., ET AL. (2002). Working memory for complex figures: An fMRI comparison of letter and fractal $n$-back tasks. Neuropsychology, 16, 370-379.

Ravizza, S. M., BehrmanN, M., \& Fiez, J. A. (2005). Right parietal contributions to verbal working memory: Spatial or executive? Neuropsychologia, 43, 2057-2067.

Spence, C., Pavani, F., Maravita, A., \& Holmes, N. (2004). Multisensory contributions to the 3-D representation of visuotactile peripersonal space in humans: Evidence from the crossmodal congruency task. Journal of Physiology, 98, 171-189.

Verdejo-Garcia,A.,Bechara,A.,Recknor,E.C., \& Perez-Garcia,M. (2006). Executive dysfunction in substance dependent individuals during drug use and abstinence: An examination of the behavioral, cognitive and emotional correlates of addiction. Journal of the International Neuropsychological Society, 12, 405-415.

VERRILLO, R.T.(1963). Effect of contactor area on the vibrotactile threshold. Journal of the Acoustical Society of America, 35, 1962-1966.

(Manuscript received January 14, 2007; revision accepted for publication June 25,2007 .) 